\title{
Long-Term Risperidone Treatment Induces Visceral Adiposity Associated with Hepatic Steatosis in Mice: A Magnetic Resonance Approach
}

\author{
Florent Auger, ${ }^{1,2,3,4}$ Patrick Duriez, ${ }^{1,2,4,5}$ Françoise Martin-Nizard, 1,5,6,7 \\ Nicolas Durieux, ${ }^{1,4}$ Régis Bordet, ${ }^{1,2,4}$ and Olivier Pétrault ${ }^{1,4,8,9}$ \\ ${ }^{1}$ Université Lille Nord de France, 59000 Lille, France \\ ${ }^{2}$ Département de Pharmacologie Médicale, CHULille, EA 1046, 59000 Lille, France \\ ${ }^{3}$ Inserm, U837, 59000 Lille, France \\ ${ }^{4}$ IMPRT-IFR114, 59000 Lille, France \\ ${ }^{5}$ Faculté de Pharmacie, 59006 Lille, France \\ ${ }^{6}$ Inserm, U1011, 59000 Lille, France \\ ${ }^{7}$ Institut Pasteur de Lille, 59019 Lille, France \\ ${ }^{8}$ UArtois, 62300 Lens, France \\ ${ }^{9}$ Laboratoire de Pharmacologie Médicale, EA 1046, Faculté de Médecine, Université Lille2, Pôle Pecherche, 1 Place de Verdun, \\ 59045 Lille Cedex, France
}

Correspondence should be addressed to Olivier Pétrault; olivier.petrault@univ-artois.fr

Received 28 July 2013; Accepted 3 April 2014; Published 27 April 2014

Academic Editor: Sonia Dollfus

Copyright (C) 2014 Florent Auger et al. This is an open access article distributed under the Creative Commons Attribution License, which permits unrestricted use, distribution, and reproduction in any medium, provided the original work is properly cited.

\begin{abstract}
Although atypical antipsychotic drugs (APDs) have led to significant advances in the treatment of psychotic disorders, they still induce metabolic disturbances. We aimed at characterizing the metabolic consequences of a risperidone treatment and at establishing a link with noninvasive MR markers, in order to develop a tool for predicting symptoms of the metabolic syndrome. Fat deposition and liver morphometry were assessed by T1-weighted imaging. Fatty acid composition and fat accumulations in tissues were determined using MR spectroscopy with and without water suppression, respectively. Risperidone treatment induced a weight gain accompanied with metabolic disturbances such as hyperglycemic status, an increase in visceral adipose tissue (VAT), and liver fat depositions. Correlations using Methylene-Water Ratio (MWR) and Polyunsaturated Index (PUI) demonstrated a concomitant increase in the weight gain, VAT and liver fat depositions, and a decrease in the quantity of polyunsaturated fatty acids. These results were consistent with a hepatic steatosis state. We evaluated the ability of MR techniques to detect subtle metabolic disorders induced by APDs. Thus, our model and methodology offer the possibility to investigate APDs side effects in order to improve the health conditions of schizophrenic patients.
\end{abstract}

\section{Introduction}

Antipsychotic drugs (APDs) are widely used in current psychiatric practice and are commonly classified as typical (conventional) or atypical (second generation). Atypical APDs have been introduced in clinical practice after 1990, including clozapine, olanzapine, quetiapine, and risperidone. Atypical APDs cause less extrapyramidal symptoms (tremors) than typical APDs. However, both of them produce a weight gain [1-3], which increases the risk to develop a metabolic syndrome [4] associating several disorders such as diabetes mellitus, hypertension, hyperglycemia, dyslipidemia, and abdominal fat deposition $[4,5]$.

The excess of visceral adipose tissue (VAT) mass is particularly correlated to the prevalence of metabolic syndrome and insulin resistance [6]. Indeed, abnormal VAT depositions lead to the storage of lipids in undesired organs such as pancreas, skeletal muscle, heart, and liver. This so-called 
"ectopic fat deposition" contributes to the development of metabolic syndrome [6-12]. VAT accumulation could thus represent a biomarker of metabolic disturbances, as used in clinic through the measurement of waist circumference in replacement of the body mass index [5]. Nevertheless, the susceptibility to develop a metabolic syndrome is not systematically linked to risk factors as fat distribution in other compartments (i.e., visceral versus subcutaneous fat), insulin-resistance, or sedentary lifestyle [7-11]. The relative independence of these factors points out the difficulty to find reliable biomarkers. "Ectopic fat deposition" remains the most relevant process for diagnosing early metabolic disturbances, thus justifying the use of new noninvasive techniques to characterize body fat repartition.

Several techniques are widely used for the in vivo assessment of body fat, such as anthropometry [13], hydrodensitometry, air displacement plethysmography, bioelectric impedance, and dual energy X-ray absorptiometry [14, 15]. Their common limitation arises from their inefficiency in distinguishing subcutaneous adipose tissue (SAT) from VAT and in establishing an accurate body fat repartition. Computerized Tomography (CT) and Magnetic Resonance (MR) scans have the capability to distinguish SAT from VAT [1621]. CT and MR techniques enable quantification of ectopic fat $[16,22-26]$. MR imaging (MRI) and Magnetic Resonance Spectroscopy (MRS) are noninvasive techniques which are suitable for fat quantification and fat nature identification [27]. T1-weighted images and Dixon sequence are usually recorded to quantify SAT and VAT [7, 19, 26, 28]. They allow the segmentation of adipose tissues by dedicated posttreatment software $[17,29]$. MRS enables the in vivo hepatic triglyceride (TG) quantification in order to diagnose hepatic steatosis in clinical practice. Due to its good correlation with histological data from liver biopsies [30], MRS is now considered as a reference for the quantification of noninvasive hepatic fat $[7,28]$.

Previous work showed the relevance of a mouse model for assessing weight gain induced by risperidone treatment [31]. In the present study, and for a translational purpose, we aimed at: (i) characterizing the metabolic disturbances associated with the weight gain induced by a long-term risperidone treatment and (ii) establishing a link with noninvasive MR markers, in order to develop a tool for predicting symptoms of the metabolic syndrome.

\section{Materials and Methods}

2.1. Animals. The Ethic community approved all protocols for animal experiments. Twelve seven-week- old female mice of C57BL/6N strain were purchased from Charles River laboratories (L'Arbresle, France).

2.2. Drug Treatment. Twenty-four female mice were examined in this study. Two groups of twelve animals were randomized by weight. One group was treated weekly over 24 weeks with intraperitoneal (IP) injection of $12.5 \mathrm{mg} \mathrm{kg}^{-1}$ of long-acting risperidone (Risperdal Consta, Janssen, USA) (risperidone group). The other group received IP injections of the vehicle of long-acting risperidone (control group). Mice were housed in a cage and maintained under a $12 \mathrm{~h}$ light-12 h dark cycle with free access to food and water. MR examinations were performed on the twenty-four mice. Pelleted food was weighted weekly to assess total grams of food consumed per cage and per day, and then divided by six to estimate the average weekly food consumption per mouse. Following MR examinations, the twenty-four mice were sacrificed by an overdose of pentobarbital. Biological samples were collected for histological examination.

2.3. MRI. All MR examinations were done after the 24 weeks of risperidone treatment on a $7 \mathrm{~T}$ Bruker Biospec (Ettlingen, Germany) imaging system equipped with a $40 \mathrm{~cm}$ horizontal bore magnet. Anesthesia was induced by $2 \%$ isoflurane and maintained at $1 \%-1.5 \%$ along acquisition, depending on respiration frequency. A pneumatic pillow (SA Inc. Stony Brook, NY) was used to perform and monitor triggered respiratory gating. Each mouse was placed in a cylindrical coil (39 mm inner diameter). Multislice gradient echo sequence was triggered with respiration and performed to assess mouse position inside the magnet, with the following sequence parameters: TR/TE (Repetition Time/Echo Time $)=$ $200 / 3 \mathrm{~ms}$, FA (Flip Angle) $=30^{\circ}$.

Liver and adipose tissue images were acquired by T1weighted imaging, using axial and coronal Rapid Acquisition with Relaxation Enhancement (RARE) sequence, which was gated with respiration. Multislice coronal $(\mathrm{FOV}=3.6 \times$ $3.6 \mathrm{~cm}, 14$ contiguous slices of $1.5 \mathrm{~mm}$ thick) and axial (FOV $=3 \times 3 \mathrm{~cm}, 18$ contiguous slices of $2 \mathrm{~mm}$ thick) RARE images were collected with a TR of $400 \mathrm{~ms}$, a TE of $9 \mathrm{~ms}$, a $256 \times 256$ data matrix, and a number of repetitions (NEX) of 2. Reconstructions were done to evaluate the volumes of liver, Inguinal Adipose Tissue (IAT, characterizing the subcutaneous compartment), and VAT (defined as the sum of perirenal fat, gonadal fat, and mesenteric fat, Figure 1(a)). Reconstructions were manually drawn on ITKSnap software [32] for each set of T1-weighted images.

2.4. MRS. Single-voxel localized $1 \mathrm{H}$ MR spectra were acquired using the PRESS sequence (respiratory gated) with the following parameters: voxel volume $3 \times 3 \times 3 \mathrm{~mm}$; TR = $10,000 \mathrm{~ms} ; \mathrm{TE}=20 \mathrm{~ms}$. To avoid obvious blood vessel and subcutaneous fat contributions, the voxel was carefully placed on previous anatomic axial and coronal T1-weighted images (Figure 1(b)). The magnetic field homogenization remains a critical step, and the local shimming was performed until the water line width achieves less than $50 \mathrm{~Hz}$ conditioning the spectral resolution. Spectral data were processed on Topspin 2.0 (Bruker, Germany).

2.5. Methyl Water Ratio (MWR). The MWR was assessed from localized proton spectroscopy performed without water suppression. In vivo, hepatic fatty acid proportion is determined as the percentage of the bulk methylene resonance to water [33]. So, MWR is defined as the ratio of the intensity of the lipid proton peak (methylene bulk) with the intensity of 


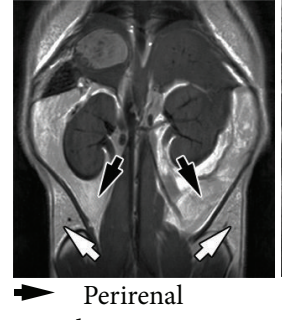

adipose tissue

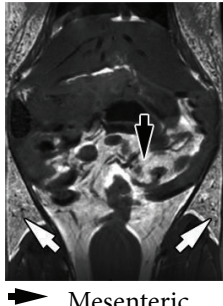

Mesenteric
adipose tissue



adipose tissue

$\approx$ Inguinal adipose tissue

(a) Voxel position

Voxel position for spectroscopy

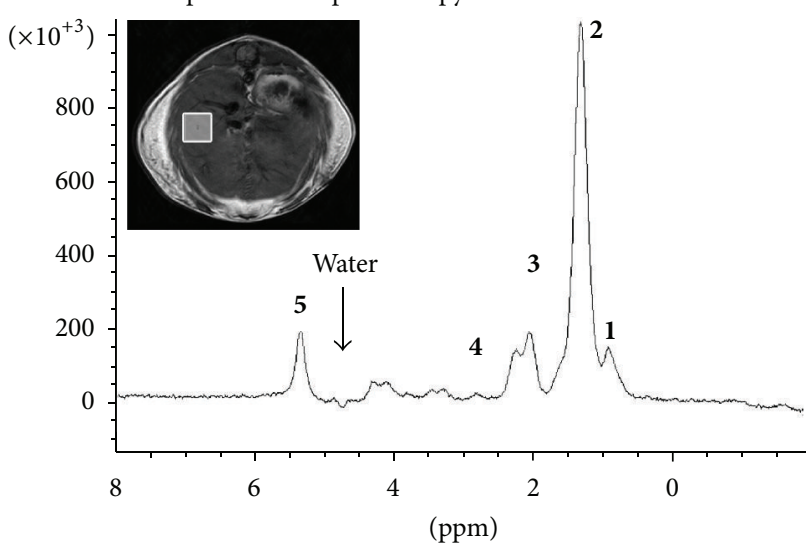

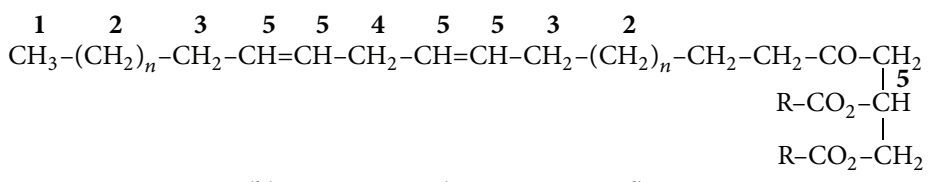

(b) Hepatic MRS (water suppressed)

Figure 1: Hepatic lipids composition. (a) Three coronal T1-weighted MR images displaying inguinal, perirenal, mesenteric, and gonadal adipose tissues. These images were used to perform volumetric analyses. (b) Axial T1-weighted liver image with the voxel position used for MRS analyses and an example of a water suppressed MRS spectrum. Each peak corresponds to a particular chemical group described as follows: $\mathbf{1}=$ terminal methyl $\left(\mathrm{CH}_{3}\right)$ at $0.9 \mathrm{ppm} ; \mathbf{2}=$ methylene groups of saturated fatty acids and the "saturated" components of mono- and poly fatty acids $\left(\mathrm{CH}_{2}\right)_{n}$ at $1.3 \mathrm{ppm} ; \mathbf{3}=$ allylic as the methylene adjacent to methene groups $\left(\mathrm{CH}_{2}\right)$ at $2.1 \mathrm{ppm} ; \mathbf{4}=$ diallylic as the methylene inserted between sequential methene groups of fatty acid $\left(\mathrm{CH}-\mathrm{CH}_{2}-\mathrm{CH}\right)$ at $2.8 \mathrm{ppm} ; \mathbf{5}=$ methene as the olefinic groups $(\mathrm{CH}=\mathrm{CH})$ at $5.3 \mathrm{ppm}$.

the water proton peak, allowing the estimation of the quantity of intracellular fat accumulation in the tissue.

2.6. Lipid MR Spectroscopy. The localized proton spectroscopy was acquired with manual water suppression using VAPOR (VAriable Power and Optimized Relaxation delays) sequence module and a signal accumulation amounted to 64. The different ratios were calculated from peak intensities of the lipid spectra. Previous works reported that the T1 relaxation values of liver methylene and methene groups were respectively between 0.39 to $1.20 \mathrm{~s}$ and 0.60 to $1.16 \mathrm{~s}[34,35]$. In order to fully relax all resonances (up to $5 \times \mathrm{T} 1$ ), we set the TR parameter of the PRESS sequence to $10 \mathrm{~s}$ and no further T1 correction was necessary. Depending on spectral resolution, the MR lipid spectrum profile is characterized by nine peaks but only five of them were usually considered for the lipid index calculation (see Figure 1(b)): $\mathbf{1}=$ terminal methyl $\left(\mathrm{CH}_{3}\right)$ at $0.9 \mathrm{ppm} ; 2=$ methylene groups of saturated fatty acids and the "saturated" components of mono- and poly fatty acids $\left(\mathrm{CH}_{2}\right)_{n}$ at $1.3 \mathrm{ppm} ; \mathbf{3}=$ allylic as the methylene adjacent to methene groups $\left(\mathrm{CH}_{2}\right)$ at $2.1 \mathrm{ppm} ; \mathbf{4}=$ diallylic as the methylene inserted between sequential methene groups of fatty acid $\left(\mathrm{CH}-\mathrm{CH}_{2}-\mathrm{CH}\right)$ at $2.8 \mathrm{ppm} ; 5=$ methene as the olefinic groups $(\mathrm{CH}=\mathrm{CH})$ at $5.3 \mathrm{ppm}$.

Calculation of hepatic lipid indexes was based on the proportion of constitutive saturated mono- and poly unsaturated fatty acids according to Johnson and colleagues' study [36]. Using MR spectroscopy, four indexes can be calculated: the unsaturated index (UI), the unsaturated index surrogate (UIs), the saturated index (SI), and the polyunsaturated index (PUI). The formulas were as follows:

$$
\mathrm{UI}=\frac{I_{\text {methene }}}{I_{\text {methene }}+I_{\text {allylic }}+I_{\text {methylene }}+I_{\text {methyl }}},
$$


where $I_{\text {methene }}, I_{\text {allylic }}, I_{\text {methylene }}$, and $I_{\text {methyl }}$ are, respectively, the signal amplitude of the methene, allylic methylene, bulk methylene, and terminal methyl peaks.

UIs formula is based on the principle that methene functional group is always next to allylic methylene group in monounsaturated fatty acids and with both allylic and diallylic methylene groups in Poly Unsaturated Fatty Acids (PUFAs). Thus UIs formula is as follows:

$$
\mathrm{UIs}=\frac{I_{\text {allylic }}+I_{\text {diallylic }}}{I_{\text {allylic }}+I_{\text {diallylic }}+I_{\text {methylene }}+I_{\text {methyl }}},
$$

where $I_{\text {diallylic }}$ is the signal amplitude of the diallylic methylene peak. SI is calculated as the complemented index of $\mathrm{UI}_{\mathrm{s}}$ and can be easily assessed as follows:

$$
\mathrm{SI}=1-\frac{I_{\text {allylic }}+I_{\text {diallylic }}}{I_{\text {allylic }}+I_{\text {diallylic }}+I_{\text {methylene }}+I_{\text {methyl }}} .
$$

Lastly, the diallylic peak is detected only in PUFAs $(18: 2$, $18: 3,20: 4,20: 5$, and $22: 6)$ in liver. Thus the measure of PUI is

$$
\text { PUI }=\frac{I_{\text {diallylic }}}{I_{\text {allylic }}+I_{\text {diallylic }}+I_{\text {methylene }}+I_{\text {methyl }}} .
$$

As the methene signal could not be correctly detected when water suppression pulses are applied, we decided to omit UI according to the method described by Johnson and colleagues [36] and the unsaturated index was therefore calculated with UIs. A spectrum with a low signal noise ratio $(<2)$ for the diallylic peak at $2.8 \mathrm{ppm}$ was excluded from the study.

2.7. Spontaneous Locomotor Activity. Spontaneous motor activity was measured using an actimeter (Panlab, Barcelona, Spain). This apparatus allowed horizontal motor activity (traveled distance, in centimeters), rearing behavior (number of rears), and mean velocity (in centimeters per second). These parameters gave clues about ability of mice to explore a new environment. Motor activity was recorded for ten minutes.

2.8. Glucose Tolerance Test. After fasting in fresh cages for 6 hours, approximately $25 \mu \mathrm{L}$ of tail tip blood was collected to assess fasting glucose levels. Thereafter, mice received $2 \mathrm{~g} / \mathrm{kg}$ of $15 \%$ dextrose by ip injection. Sample tail tip blood glucose levels were measured using a glucometer (AccuCheck performa, Roche Diagnostics $\mathrm{GmbH}$, Mannheim, Germany) at 10, 20, 30, 60, 90, and $120 \mathrm{~min}$.

2.9. Histology. IAT and livers were collected and weighted. Livers were then fixed in $4 \%$ paraformaldehyde for Oil Red $\mathrm{O}$ (ORO) staining. Analysis of lipid deposition was made by ORO staining on $7 \mu \mathrm{m}$-thick frozen-liver sections, using Harris haematoxylin for nucleus coloration. The surface of liver lipid droplets was quantified using image J software (1.43, Wayne Rasband, NIH, USA). Lipid droplet volumes were calculated from the surface previously measured by the software. Results were presented as a volume variation relative to the control group.

\subsection{Assays}

2.10.1. Plasma. Dosage of cholesterol and TG was performed immediately after MR. Plasma levels of total cholesterol (TC), TGs, and high density lipoprotein cholesterol (HDLc) were determined using commercially available kits (BioMérieux, France). Non-HDL cholesterol (Non-HDLc) was calculated by subtraction of HDLc from TC.

2.10.2. Livers TG. Frozen liver tissue $(50 \mathrm{mg})$ was homogenized in SET buffer ( $1 \mathrm{~mL}$; sucrose $250 \mathrm{mM}$, EDTA $2 \mathrm{mM}$, and Tris $10 \mathrm{mM}$ ), followed by two freeze-thaw cycles and three times passages through a 27-gauge syringe needle, and a final freeze-thaw cycle. Protein content was determined using the BCA method (Interchim, France), and TG and cholesterol were measured as described above.

2.11. Statistical Analyses. All statistical analyses were performed using StatWiev software (SAS institute Inc., Version 5.0). Data are reported as mean \pm standard deviation (SD). The criterion for statistical significance was $P<0.05$.

Statistical analyses were performed as follows.

(1) When distributions were normal in each group, a Student's $t$-test was used. If one or more group was not normally distributed, the nonparametric MannWhitney was used.

(2) To assess differences on autopsy, MR volumetry, and MRS experimental datasets, we performed ANCOVA tests using mice weight as a covariate in order to adjust statistical analyses and suppress the influence of the weight gain.

(3) For correlations among data, a Spearman test was used.

(4) A two-way ANOVA was performed to test weight gain and food intake.

\section{Results}

3.1. Risperidone Induced a Long-Term Weight Gain in Mice. After 24 weeks, a greater weight gain was observed in mice treated by risperidone compared to the control mice. At the beginning of the experiment, the mean weight of the risperidone group was $16.75 \pm 0.87 \mathrm{~g}$ and $16.00 \pm 1.04 \mathrm{~g}$ for the control group to achieve, respectively, $31.33 \pm 2.42 \mathrm{~g}$ versus $24.58 \pm 1.21 \mathrm{~g}(P<0.01, n=24)$ at the end of the experiment (Figure 2(a)). In addition, this weight gain was associated with a change in food intake in treated animals from the 13th week (Control: $3.90 \pm 0.17 \mathrm{~g} /$ day/mouse versus Risperidone: $4.43 \pm 0.47 \mathrm{~g} /$ day $/$ mouse; $P<0.05 ; n=24$ Figure $2(\mathrm{~b})$ ) as well as with a modification in spontaneous locomotor activity (Table 1). Indeed, the treatment induced a reduction of the distance covered in the arena (Control: 6, $300 \pm 687 \mathrm{~cm}$ versus Risperidone: $5,041 \pm 688 \mathrm{~cm} P<0.001 ; n=24$; Table 1), in association with a diminution of the mean velocity of animals (Control: $12.11 \pm 1.55 \mathrm{~cm} \cdot \mathrm{s}^{-1}$ versus Risperidone: $9.54 \pm 1.27 \mathrm{~cm} \cdot \mathrm{s}^{-1} ; \quad P<0.01, n=24$; Table 1$)$. Moreover, the exploration behaviour was also modified by the treatment 
as revealed by the reduction of the rearing number (Control: $29.17 \pm 10.07$ versus Risperidone: $65.83 \pm 11.68 ; P<0.01$, $n=24$; Table 1). These results suggest that the risperidoneinduced weight gain could at least in part be linked to an increase of appetite combined with a reduced spontaneous locomotor activity.

\subsection{Could the Risperidone-Induced Weight Gain Be Associated} with Metabolic Disturbances? We sought several metabolic disorders such as glycemic status, blood lipid analysis, and specific biological samples as the liver and fat depositions in order to identify peripheral consequences of the weight gain induced risperidone. The glucose tolerance test revealed that risperidone led to a hyperglycemic status as shown by the glucose tolerance test (Figure 3(a)) and by the area under the curve values (AUC: $27453 \pm 2389(\mathrm{mg} / \mathrm{dL} \cdot 120 \mathrm{~min})$ versus $42276 \pm 12999$ (mg/dL·120 min); $P<0.01, n=20$; Table 2$)$. Blood analysis showed that risperidone increased the nonHDLc level compared to control mice $(26.90 \pm 6.73 \mathrm{mg} / \mathrm{dL}$ versus $18.82 \pm 7.61 \mathrm{mg} / \mathrm{dL} ; P<0.01, n=24)$. But no significant difference was found concerning fasting TG $(78.07 \pm 30.07 \mathrm{mg} / \mathrm{dL}$ versus $66.21 \pm 11.18 \mathrm{mg} / \mathrm{dL}, P=0.05637$, $n=24)$ and HDLc concentrations $(44.20 \pm 6.77 \mathrm{mg} / \mathrm{dL}$ versus $42.59 \pm 5.121 \mathrm{mg} / \mathrm{dL}, P=0.8625, n=24$; Figure $3(\mathrm{~b})$ ).

To test whether the risperidone treatment modified the fat deposition, we quantified subcutaneous inguinal and visceral fat from anatomic MR images as defined previously (Figure 1). Table 2 summarizes the volumetric analyses. The IAT and VAT volumes were, respectively, 2-fold and 3-fold higher in risperidone-treated mice than in the control group (IAT: $544.19 \pm 127.20 \mathrm{~mm}^{3}$ versus $232.40 \pm 34.20 \mathrm{~mm}^{3}$ and VAT: $1065.06 \pm 383.76 \mathrm{~mm}^{3}$ versus $317.89 \pm 116.79 \mathrm{~mm}^{3}$; $P<0.001, n=24$; Table 2). In addition, the best correlation between the adipose tissue accumulation and the glucose tolerance test was interestingly found for the VAT (correlation factor $=0.867, P<0.001, n=20$; Table 3 ), suggesting that VAT could represent a good biomarker of the hyperglycemic status in our experimental model.

At the liver level, the autopsies revealed that risperidone treatment increased the liver weight $(1.50 \pm 0.17 \mathrm{~g}$ versus $1.10 \pm 0.14 \mathrm{~g} ; P<0.001, n=24$; Table 2$)$. This hepatomegaly was confirmed by the volumetric data from MRI, as demonstrated by the significant increase in liver volume after risperidone treatment $\left(702.77 \pm 32.01 \mathrm{~mm}^{3}\right.$ versus $635.74 \pm 49.54 \mathrm{~mm}^{3} ; P<0.01, n=24$; Table 2 ). The ORO staining quantification showed a significantly higher lipid accumulation in the liver of mice treated by risperidone than in the control mice $(100.00 \pm 46.58 \%$ versus $210.96 \pm$ 68.19\%; $P<0.001, n=21$; Figure 3(c), left panel). Results from hepatic lipid analyses were expressed as the ratio of TG to the protein concentration. The risperidone treatment increased the TG/protein ratio, pointing out an intrahepatic accumulation of TG $(0.327 \pm 0.096$ versus $0.225 \pm 0.097$; $P<0.05, n=24$; Figure 3(c), right panel). This histological pattern clearly shows an early hepatic steatosis.

3.3. Quantification of the Hepatic Fat by MR Spectroscopy. Spectroscopy can easily distinguish the quantities of water and fat in body tissues. This technique is based on the calculation of the methylene/water ratio (MWR; Figure 4(a)). The MWR values calculated from hepatic spectra were significantly higher in the risperidone-treated group than in the control group $(3.57 \pm 1.26 \%$ versus $1.10 \pm 0.20 \%$; $P<$ $0.001, n=24$; Figure 4(a), histogram). This result was in good accordance with the histological pattern, pointing out the high sensitivity of the MRS technique for the detection of fat accumulation in the liver. We drew a correlation between the MWR/TG protein ratio (correlation factor $=0.798, P<$ $0.001, n=24$; Table 3 ) and ORO staining proportion (correlation factor $=0.747, P<0.01, n=20$; Table 3), also found proportional to the fat accumulation in the liver.

Table 3 summarizes the link between MRS, MRI, and the postmortem data. We found a strong correlation between $\mathrm{MWR} / \mathrm{VAT}$ volume (correlation factor $=0.798, P<0.001$, $n=24$; Table 3 ) and MWR/mice weight (correlation factor $=0.859, P<0.001, n=24$; Table 3). These correlations demonstrated a concomitant increase of weight gain, fat accumulation in the liver, and VAT deposition.

3.4. Qualitative Aspects of the Lipid MR Spectrum. Given the limit of $50 \mathrm{~Hz}$ for spectral resolution, 4 acquisitions had not been performed, 2 in each group. Therefore, 20 animals were considered in lipid indexes calculations described previously in the methods section and in Figure 1(b). In addition to the liver fat accumulation, the spectral analysis provides information on the realignment of the lipid chains. Compared to the control group, the risperidone treatment had an effect on UIs (Risperidone: $0.156 \pm 0.016$ versus Control: $0.182 \pm 0.021 ; P<$ $0.01, n=20$ ), on SI (Risperidone: $0.844 \pm 0.016$ versus Control: $0.818 \pm 0.021 ; P<0.05, n=20$ ), and on PUI (Risperidone: $0.017 \pm 0.008$ versus Control: $0.047 \pm 0.023 ; P<0.001$, $n=20$ ) as illustrated in Figure 4(b). The decrease in PUI and the concomitant increase in SI suggest a pathological state of the fatty acid availability in a suffering liver. The aggravation of hepatic steatosis was proportional to the decrease in PUI values. In our model, we found a closed correlation between PUI and mice weight (correlation factor $=-0.868, P<0.001$, $n=20$; Table 3).

\section{Discussion}

Two aspects are addressed in this study: first, our experimental model, which gradually developed a metabolic disturbance pattern associated with a long-term risperidone treatment, and second, our methodology, based on magnetic resonance, which evidenced that these noninvasive techniques were suitable for the detection of body fat alterations and the resulting hepatic steatosis.

In our experimental mouse model, the risperidone treatment induced a weight gain after 24 weeks. We concomitantly described here components of the metabolic syndrome [5] in mice treated by risperidone. Treated mice developed (i) a hyperglycemic status, (ii) an increase in non-HDL cholesterol plasma level, (iii) an abnormal deposition of fat in the inguinal and visceral areas, and (iv) a hepatomegaly related with early hepatic steatosis. The other advantages 
TABLE 1: Spontaneous locomotor activity.

\begin{tabular}{lccccc}
\hline \multirow{2}{*}{ Data sets } & \multicolumn{2}{c}{ Control group } & \multicolumn{2}{c}{ Risperidone group } \\
& $n$ & Mean $\pm \mathrm{SD}$ & $n$ & Mean \pm SD & Significance \\
\hline Distance $(\mathrm{cm})$ & 12 & $6300 \pm 687$ & 12 & $5041 \pm 688$ & $\mathrm{~S}(P<0.001)$ \\
Mean velocity $\left(\mathrm{cm} \cdot \mathrm{s}^{-1}\right)$ & 12 & $12.11 \pm 1.55$ & 12 & $9.54 \pm 1.27$ & $\mathrm{~S}(P<0.001)$ \\
Numbers of rearing & 12 & $65.83 \pm 11.68$ & 12 & $29.17 \pm 10.07$ & $\mathrm{~S}(P<0.001)$ \\
\hline
\end{tabular}



- Risperidone

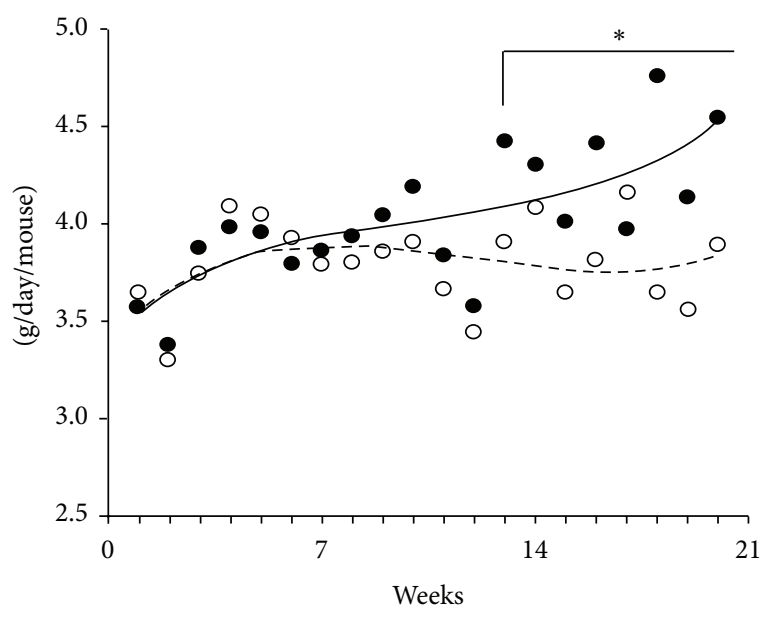

O Control

- Risperidone

(a) Weight gain

(b) Food intake

FIGURE 2: Twenty-four week risperidone treatment increased mice weight and food intake. (a) Effect of risperidone on weight gain in female mice. Differences between both groups appeared significant after 7 weeks of treatment and remained significant at the end of the experiment (24 weeks: control (॰) $24.58 \pm 1.21 \mathrm{~g}$ versus risperidone $(\bullet) 31.33 \pm 2.42 \mathrm{~g} ; P<0.01, n=24$ ). (b) Effect of risperidone on food intake. Risperidone treatment increased food intake after 13 weeks (Control: $3.90 \pm 0.17 \mathrm{~g} /$ day/mouse versus Risperidone: $4.43 \pm 0.47 \mathrm{~g} / \mathrm{day} / \mathrm{mouse}$ $P<0.05 ; n=24)$.

TABLE 2: Experimental parameters analysis.

\begin{tabular}{|c|c|c|c|c|c|c|}
\hline \multirow{2}{*}{ Data Sets } & & \multicolumn{2}{|c|}{ Control group } & \multicolumn{2}{|c|}{ Risperidone group } & \multirow{2}{*}{ Significance } \\
\hline & & $n$ & Mean \pm SD & $n$ & Mean \pm SD & \\
\hline $\mathrm{AUC}(\mathrm{mg} / \mathrm{dL} \cdot 120 \mathrm{~min})$ & & 10 & $27,453 \pm 2,389$ & 10 & $42,276 \pm 12,999$ & $\mathrm{~S}(P<0.001)$ \\
\hline Tissue weight $(\mathrm{g})$ & Liver & 12 & $1.10 \pm 0.14$ & 12 & $1.50 \pm 0.17$ & $\mathrm{~S}(P<0.001)$ \\
\hline \multirow{3}{*}{ Tissue volume $\left(\mathrm{mm}^{3}\right)$} & Liver & 12 & $635.74 \pm 49.54$ & 12 & $702.77 \pm 32.01$ & $\mathrm{~S}(P<0.01)$ \\
\hline & IAT & 12 & $232.40 \pm 34.20$ & 12 & $544.19 \pm 127.20$ & $\mathrm{~S}(P<0.001)$ \\
\hline & VAT & 12 & $317.89 \pm 116.78$ & 12 & $1,065.06 \pm 383.76$ & $\mathrm{~S}(P<0.001)$ \\
\hline
\end{tabular}

TABLE 3: Correlations.

\begin{tabular}{|c|c|c|c|c|c|}
\hline Correlations & & $n$ & $R^{2}$ & Correlation factor & Significance \\
\hline \multirow{3}{*}{ AUC/tissue volume } & Liver & 20 & 0.080 & 0.406 & NS \\
\hline & VAT & 20 & 0.349 & 0.867 & $\mathrm{~S}(P<0.001)$ \\
\hline & IAT & 20 & 0.269 & 0.772 & $\mathrm{~S}(P<0.001)$ \\
\hline \multirow{2}{*}{ MWR/hepatic lipid assay } & TG/protein & 24 & 0.440 & 0.798 & $\mathrm{~S}(P<0.001)$ \\
\hline & ORO staining & 20 & 0.695 & 0.747 & $\mathrm{~S}(P<0.01)$ \\
\hline MWR/VAT & & 24 & 0.610 & 0.798 & $\mathrm{~S}(P<0.001)$ \\
\hline MWR/mice weight & & 24 & 0.651 & 0.859 & $\mathrm{~S}(P<0.001)$ \\
\hline MWR/AUC & & 20 & 0.611 & 0.798 & $\mathrm{~S}(P<0.001)$ \\
\hline PUI/mice weight & & 20 & 0.399 & -0.868 & $\mathrm{~S}(P<0.001)$ \\
\hline
\end{tabular}




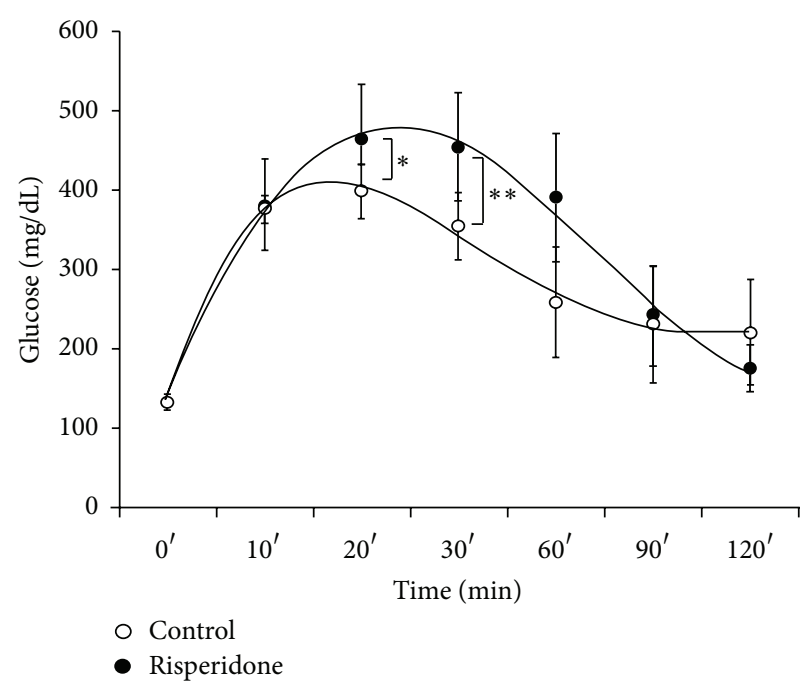

(a) Glucose tolerance

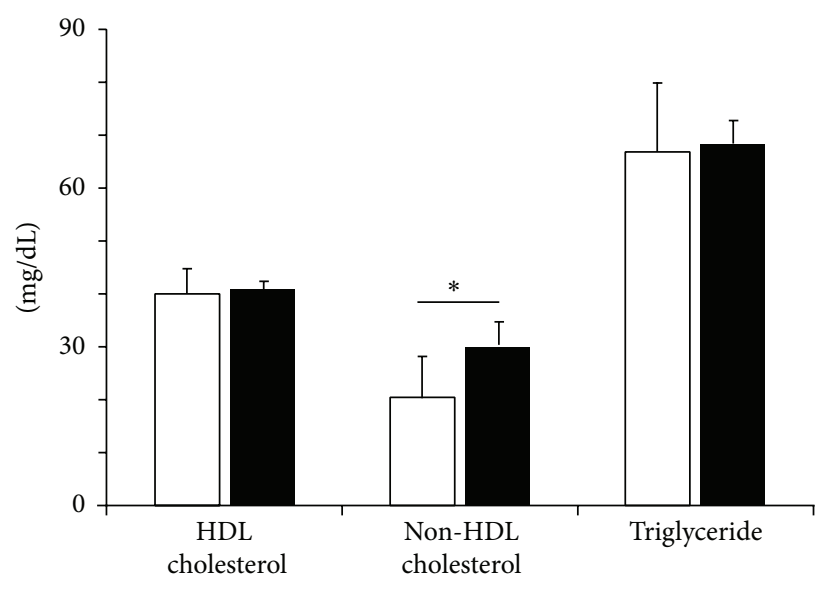

$\square$ Control

- Risperidone
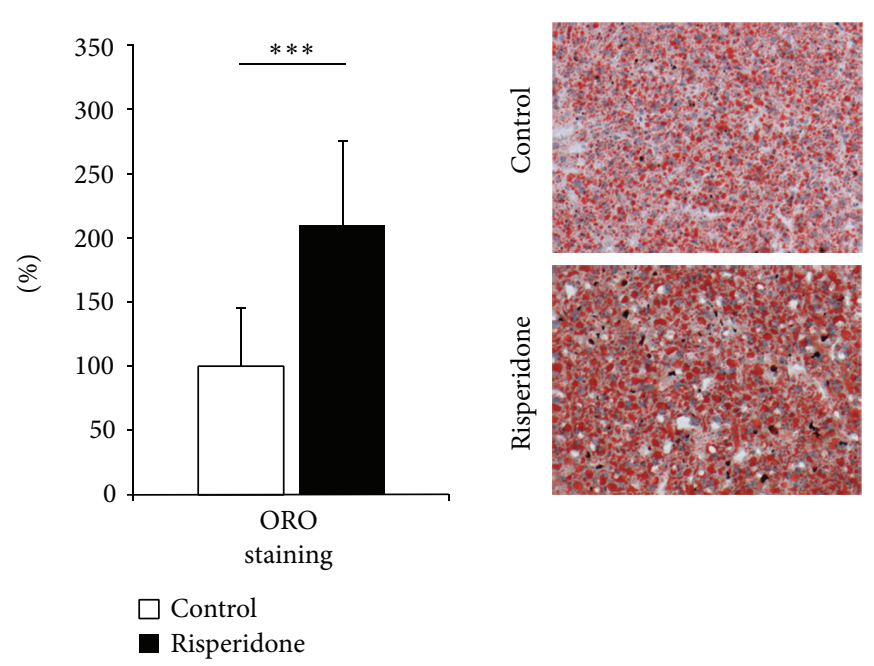

(b) Plasma lipid

(c) Liver lipid

Figure 3: Metabolic disturbances induced by a 24-week treatment of risperidone on female mice. (a) Effect of risperidone on glucose tolerance in female mice. Risperidone treatment significantly decreased glucose tolerance. (b) Effect of risperidone on plasma lipid concentration. Risperidone treatment increased non-HDLc $(26.90 \pm 6.73 \mathrm{mg} / \mathrm{dL}$ versus $18.82 \pm 7.61 \mathrm{mg} / \mathrm{dL} ; P<0.01, n=24)$. (c) Effect of risperidone on liver fat accumulation. Risperidone treatment induced an increase in liver lipid accumulation as displayed by ORO staining (control ( $\square$ ) $100.00 \pm 46.58 \%$ versus risperidone ( $\square$ ) $210.96 \pm 68.19 \%$; $P<0.05, n=21$; left histogram and central pictures) and TG/protein ratio (control () $0.225 \pm 0.097$ versus risperidone $(\square) 0.327 \pm 0.096 ; P<0.05, n=24$; right histogram).

of this model are based on the risperidone-induced change in behaviour of the animal in terms of food intake and spontaneous locomotor activity. Several pieces of evidence support the idea that ADPs-induced weight gain would result from an increase in appetite self-mediated by a histaminic pathway at the CNS level [37]. Furthermore, risperidone could reduce locomotor activity by blocking the dopaminergic system $[38,39]$. All these aspects highlight the relevance of our experimental model for the study of metabolic dark-side effects of ADPs.

The second aspect of this study is the use of noninvasive MR techniques. The volumetric analysis was performed through the use of T1-weighted imaging and was allowed the quantification of fat deposition in subcutaneous and visceral areas. Our findings showed a closed correlation between VAT and hyperglycemic status, in good accordance with clinical evidences suggesting a link between the prevalence of metabolic syndrome and an abnormal visceral adiposity [6]. This is why the different components of fat had to be distinguished. Previous clinical works reported that the abnormal VAT development led to lipid storage in undesired sites such as pancreas, skeletal muscle, heart, and liver $[6,7$, 12]. In this study, we assessed the liver fat quantity reflected by MWR using MR spectroscopy and established a link between the mice being overweight, the increase in VAT, and liver fat deposition. These findings demonstrated that the MR 



Control

Risperidone

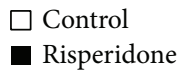

(a) Methylene/water ratio calculation


(b) Lipidic chain saturation indexes

FIGURE 4: Quantitative and qualitative in vivo lipid assessments. (a) Representative of water suppressed MR liver spectra (left; control: grey spectrum; risperidone: black spectrum). Spectra resulted from a voxel analysis positioned in liver as described in Figure 1(b). Water and methylene peaks were clearly visible and enabled the assessment of the methylene water ratio (MWR). MWR revealed that risperidone treatment increased the lipid accumulation in liver (right histogram: control ( $\square$ ) $1.10 \pm 0.20 \%$ versus risperidone (匹) $3.57 \pm 1.26 \%$; $P<$ $0.001, n=24$ ). (b) Unsaturated Index surrogate (UIs), Poly Unsaturated Index (PUI), and Saturated Index (SI) reflecting the hepatic lipid composition. Compared to control mice, risperidone significantly induced an increase in UIs (control ( $\square$ ) $0.156 \pm 0.016$ versus risperidone

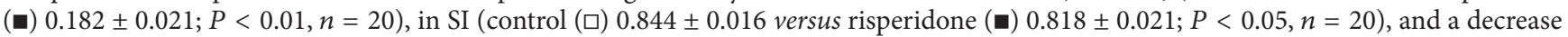
in PUI (control (口) $0.047 \pm 0.023$ versus risperidone ( $\square$ ) $0.017 \pm 0.008 ; P<0.001, n=20$ ).

methods constituted an accurate tool to study the possible fat reallocation from peripheral adipose tissue to the liver as suggested by clinical observations [40]. An interesting feature in our use of spectroscopy was the relatively low fat quantity in our model (MWR $=3.57 \pm 1.26 \%$ ) compared to other experimental models such as obese mice (ob/ob) or methionine choline deficient diet mice displaying a high fat level, respectively, $\mathrm{MWR}=38 \%$ and $\mathrm{MWR}=31 \%$ [41].

The low concentration of liver fat makes the hepatic steatosis difficult to diagnose by MR techniques. Clinical and preclinical studies showed modifications in nature and proportion of fatty acids in a suffering liver [42, 43]. The pathogenesis of steatohepatitis involves, at least in part, an oxidative process explaining the decrease in the PUFA level in steatotic liver. Indeed, assumptions indicate that polyunsaturated moieties within phospholipid membranes, the $(n-3)$ species in particular, are most susceptible to free radical attacks leading to a reallocation of unsaturations [44, 45]. Using chromatographic techniques, two major markers have been identified as relevant for characterizing steatosis: the increase in saturated fatty acids and the depletion of polyunsaturated fatty acids $[42,46]$. In our study, to validate 
the potential presence of this pathology, a water suppressed spectroscopy sequence was performed to estimate saturated and unsaturated fatty acid proportions. Compared to the control group, our spectrum analysis highlighted a significant decrease in PUI and an increase in SI in risperidone-treated mice. This result is in good agreement with previous works in which a similar variation of PUI was reported on obese patients with hepatic steatosis. This study concluded that hepatic steatosis potentiated the decrease in PUI [36]. Despite our less severe experimental model than an obesity model, our results clearly showed that the high sensitivity of the MR techniques enabled the diagnosis of early hepatic steatosis, as shown by the different correlations with PUI and MWR.

Nevertheless, these techniques have their own limitations, especially when the target organ for instance liver is subjected to respiratory motion. Although respiratory gating improves the quality of acquisitions, residual breathing artifacts may yield to uncertainty in amplitude and phase variations in localized MR spectra. These artifacts may induce bias on the spectral resolution which is dependent on the homogenization of the magnetic field (shim), and consequently modify the calculation of saturation/unsaturation indices. To minimize breathing artifacts, the ideal condition should define a TR value as close as possible to the steady state duration of breathing. Nevertheless, the use of low TR values leads to a T1-weighting at the expense of the signal/noise ratio. Compromisingly, we selected a high TR value to promote the signal/noise ratio. The magnetic field strength represents also a parameter which conditions a good signal/noise ratio, but depends only on the properties of the machine. The arrival of $3 \mathrm{~T}$ magnets for patients in clinical practice and $7 \mathrm{~T}$ magnets for clinical research may promote the use of these noninvasive techniques for diagnosis assistance through optimized signal/noise ratio and acquisition time.

Although our experimental model is far from obesity models, the subtle metabolic disturbances observed here demonstrated that MR approaches leave the opportunity to evaluate consequences of the risperidone treatment. We highlight three noninvasive markers to predict metabolic disturbances and hepatic steatosis severity when critical limits are reached and exceeded: mice weight up to $26 \mathrm{~g}$, MWR up to $2.5 \%$, and PUI down to 0.17 . As a perspective to improve health conditions of schizophrenic patients, this experimental approach (model and methodology) could represent a relevant tool to (i) define the best effective dose of ADPs to maintain CNS effects and minimize metabolic dark-side effects; (ii) evaluate the benefit of a functional food such as dietaries supplemented with omega-3 [47] or curcuminoids [48]; (iii) develop new drugs through the validation of new therapeutic strategies using an active principle or a new pharmacological adjuvant targeting the CNS or not, in a view to reduce metabolic disturbances.

\section{Conclusion}

In this study, we proposed a pathophysiologic model of slight metabolic disturbances induced by a long-term risperidone treatment, which have been evaluated in vivo by MR methods. Three parameters have been selected: MWR, PUI, and mice weight as reliable factors to define the severity of the disturbances. Thus, our model and methodology offer the possibility to investigate APDs side effects more precisely, in order to improve health conditions of schizophrenic patients.

\section{Conflict of Interests}

The authors declare that there is no conflict of interests regarding the publication of this paper.

\section{Acknowledgments}

The authors would like to thank Anthony Lucas for his technical assistance in histology, Emmanuelle Vallez for her technical contribution to chemical assays, Camille Potey for her helpful assistance in animal autopsies, and Julia Salleron for her statistical analyses contribution (Department of Biostatistic, CHU Lille, EA 2694, Univ Lille Nord de France, F-59000 Lille, France). To end, The authors would like to thank Vincent Bérézowski for his helpful assistance in the English editing of the paper. The research leading to these results has received funding from the "Conseil regional du Nord-Pas-de-Calais," the "Fond Européen de DEveloppement Régional" (FEDER), and the ministry of "Recherche et de l'Enseignement Supérieur."

\section{References}

[1] M. de Hert, J. Detraux, R. van Winkel, W. Yu, and C. U. Correll, "Metabolic and cardiovascular adverse effects associated with antipsychotic drugs," Nature Reviews Endocrinology, vol. 8, no. 2, pp. 114-126, 2012.

[2] J. A. Lieberman, T. S. Stroup, J. P. McEvoy et al., "Effectiveness of antipsychotic drugs in patients with chronic schizophrenia," The New England Journal of Medicine, vol. 353, no. 12, pp. 12091223, 2005.

[3] W. W. Fleischhacker, R. D. McQuade, R. N. Marcus, D. Archibald, R. Swanink, and W. H. Carson, "A double-blind, randomized comparative study of aripiprazole and olanzapine in patients with schizophrenia," Biological Psychiatry, vol. 65, no. 6, pp. 510-517, 2009.

[4] C. U. Correll, J. M. Kane, and P. Manu, "Obesity and coronary risk in patients treated with second-generation antipsychotics," European Archives of Psychiatry and Clinical Neuroscience, vol. 261, no. 6, pp. 417-423, 2011.

[5] K. G. M. M. Alberti and P. Zimmet, "Metabolic syndromea new world-wide definition. A consensus statement from the International Diabetes Federation," Diabetic Medicine, vol. 23, no. 5, pp. 469-480, 2006.

[6] N. Rasouli, B. Molavi, S. C. Elbein, and P. A. Kern, "Ectopic fat accumulation and metabolic syndrome," Diabetes, Obesity and Metabolism, vol. 9, no. 1, pp. 1-10, 2007.

[7] B. J. Arsenault, E. P. Beaumont, J. P. Despres, and E. Larose, "Mapping body fat distribution: a key step towards the identification of the vulnerable patient?" Annals of Medicine, vol. 44, pp. 758-772, 2011. 
[8] D. B. Carr, K. M. Utzschneider, R. L. Hull et al., "Intraabdominal fat is a major determinant of the National Cholesterol Education Program Adult Treatment Panel III criteria for the metabolic syndrome," Diabetes, vol. 53, no. 8, pp. 2087-2094, 2004.

[9] C. S. Fox, J. M. Massaro, U. Hoffmann et al., "Abdominal visceral and subcutaneous adipose tissue compartments: association with metabolic risk factors in the Framingham Heart Study," Circulation, vol. 116, no. 1, pp. 39-48, 2007.

[10] S. R. Smith, J. C. Lovejoy, F. Greenway et al., "Contributions of total body fat, abdominal subcutaneous adipose tissue compartments, and visceral adipose tissue to the metabolic complications of obesity," Metabolism, vol. 50, no. 4, pp. 425435, 2001.

[11] J. Tong, E. J. Boyko, K. M. Utzschneider et al., "Intra-abdominal fat accumulation predicts the development of the metabolic syndrome in non-diabetic Japanese-Americans," Diabetologia, vol. 50, no. 6, pp. 1156-1160, 2007.

[12] J.-P. Després and I. Lemieux, "Abdominal obesity and metabolic syndrome," Nature, vol. 444, no. 7121, pp. 881-887, 2006.

[13] A. S. Jackson and M. L. Pollock, "Steps towards the development of generalized equations for predicting body composition of adults," Canadian Journal of Applied Sport Sciences, vol. 7, no. 3, pp. 189-196, 1982.

[14] K. J. Ellis, "Human body composition: in vivo methods," Physiological Reviews, vol. 80, no. 2, pp. 649-680, 2000.

[15] S. Mattsson and B. J. Thomas, "Development of methods for body composition studies," Physics in Medicine and Biology, vol. 51, no. 13, pp. R203-R228, 2006.

[16] N. Abate, A. Garg, R. Coleman, S. M. Grundy, and R. M. Peshock, "Prediction of total subcutaneous abdominal, intraperitoneal, and retroperitoneal adipose tissue masses in men by a single axial magnetic resonance imaging slice," American Journal of Clinical Nutrition, vol. 65, no. 2, pp. 403408, 1997.

[17] J. L. Lancaster, A. A. Ghiatas, A. Alyassin, R. F. Kilcoyne, E. Bonora, and R. A. DeFronzo, "Measurement of abdominal fat with T1-weighted MR images," Journal of Magnetic Resonance Imaging, vol. 1, no. 3, pp. 363-369, 1991.

[18] J. Kullberg, J. Brandberg, J.-E. Angelhed et al., "Whole-body adipose tissue analysis: comparison of MRI, CT and dual energy X-ray absorptiometry," British Journal of Radiology, vol. 82, no. 974, pp. 123-130, 2009.

[19] J. Berglund, L. Johansson, H. Ahlström, and J. Kullberg, "Threepoint Dixon method enables whole-body water and fat imaging of obese subjects," Magnetic Resonance in Medicine, vol. 63, no. 6, pp. 1659-1668, 2010.

[20] H. Kvist, L. Sjostrom, and U. Tylen, "Adipose tissue volume determinations in women by computed tomography: technical considerations," International Journal of Obesity, vol. 10, no. 1, pp. 53-67, 1986.

[21] J. C. Seidell, C. J. G. Bakker, and K. van der Kooy, "Imaging techniques for measuring adipose-tissue distribution-a comparison between computed tomography and 1.5-T magnetic resonance," American Journal of Clinical Nutrition, vol. 51, no. 6, pp. 953-957, 1990.

[22] G. Brix, S. Heiland, M. E. Bellemann, T. Koch, and W. J. Lorenz, "MR imaging of fat-containing tissues: valuation of two quantitative imaging techniques in comparison with localized proton spectroscopy," Magnetic Resonance Imaging, vol. 11, no. 7, pp. 977-991, 1993.
[23] B. H. Goodpaster, V. A. Stenger, F. Boada et al., "Skeletal muscle lipid concentration quantified by magnetic resonance imaging," American Journal of Clinical Nutrition, vol. 79, no. 5, pp. 748754, 2004.

[24] F. Schick, J. Machann, K. Brechtel et al., "MRI of muscular fat," Magnetic Resonance in Medicine, vol. 47, no. 4, pp. 720-727, 2002.

[25] E. L. Thomas, G. Hamilton, N. Patel et al., "Hepatic triglyceride content and its relation to body adiposity: a magnetic resonance imaging and proton magnetic resonance spectroscopy study," Gut, vol. 54, no. 1, pp. 122-127, 2005.

[26] H. H. Hu, K. S. Nayak, and M. I. Goran, "Assessment of abdominal adipose tissue and organ fat content by magnetic resonance imaging," Obesity Reviews, vol. 12, no. 501, pp. e504e515, 2011.

[27] R. Ross, B. Goodpaster, D. Kelley, and F. Boada, "Magnetic resonance imaging in human body composition research. From quantitative to qualitative tissue measurement," Annals of the New York Academy of Sciences, vol. 904, pp. 12-17, 2000.

[28] S. B. Reeder, I. Cruite, G. Hamilton, and C. B. Sirlin, "Quantitative assessment of liver fat with magnetic resonance imaging and spectroscopy," Journal of Magnetic Resonance Imaging, vol. 34, no. 4, pp. 729-749, 2011.

[29] L. Wilhelm Poll, H.-J. Wittsack, J.-A. Koch et al., "A rapid and reliable semiautomated method for measurement of total abdominal fat volumes using magnetic resonance imaging," Magnetic Resonance Imaging, vol. 21, no. 6, pp. 631-636, 2003.

[30] A. P. Levene, H. Kudo, M. J. Armstrong et al., "Quantifying hepatic steatosis-more than meets the eye," Histopathology, vol. 60, no. 6, pp. 971-981, 2012.

[31] E. Lauressergues, F. Martin, A. Helleboid et al., "Overweight induced by chronic risperidone exposure is correlated with overexpression of the SREBP-1c and FAS genes in mouse liver," Naunyn-Schmiedeberg's Archives of Pharmacology, vol. 383, no. 4, pp. 423-436, 2011.

[32] P. A. Yushkevich, J. Piven, H. C. Hazlett et al., "User-guided 3D active contour segmentation of anatomical structures: significantly improved efficiency and reliability," NeuroImage, vol. 31, no. 3, pp. 1116-1128, 2006.

[33] L. S. Szczepaniak, P. Nurenberg, D. Leonard et al., "Magnetic resonance spectroscopy to measure hepatic triglyceride content: prevalence of hepatic steatosis in the general population," American Journal of Physiology-Endocrinology and Metabolism, vol. 288, no. 2, pp. E462-E468, 2005.

[34] R. Deslauriers, R. L. Somorjai, Y. Geoffrion, T. Kroft, I. C. Smith, and J. K. Saunders, "1H and 13C NMR studies of tissue from normal and diseased mice. Analysis of T1 and T2 relaxation profiles of triglycerides in liver," NMR in Biomedicine, vol. 1, no. 1, pp. 32-43, 1988.

[35] J. Ren, I. Dimitrov, A. D. Sherry, and C. R. Malloy, “Composition of adipose tissue and marrow fat in humans by $1 \mathrm{H}$ NMR at 7 Tesla," Journal of Lipid Research, vol. 49, no. 9, pp. 2055-2062, 2008.

[36] N. A. Johnson, D. W. Walton, T. Sachinwalla et al., "Noninvasive assessment of hepatic lipid composition: advancing understanding and management of fatty liver disorders," Hepatology, vol. 47, no. 5, pp. 1513-1523, 2008.

[37] S. F. Kim, A. S. Huang, A. M. Snowman, C. Teuscher, and S. H. Snyder, "Antipsychotic drug-induced weight gain mediated by histamine $\mathrm{H} 1$ receptor-linked activation of hypothalamic AMPkinase," Proceedings of the National Academy of Sciences of the United States of America, vol. 104, no. 9, pp. 3456-3459, 2007. 
[38] M. Rodríguez-Arias, I. Broseta, M. A. Aguilar, and J. Miñarro, "Lack of specific effects of selective D1 and D2 dopamine antagonists vs. risperidone on morphine-induced hyperactivity," Pharmacology Biochemistry and Behavior, vol. 66, no. 1, pp. 189-197, 2000.

[39] J. Arnt, "Differential effects of classical and newer antipsychotics on the hypermotility induced by two dose levels of Damphetamine," European Journal of Pharmacology, vol. 283, no. 1-3, pp. 55-62, 1995.

[40] H. E. Bays, "Adiposopathy: is "sick fat" a cardiovascular disease?" Journal of the American College of Cardiology, vol. 57, no. 25, pp. 2461-2473, 2011.

[41] I. R. Corbin, E. E. Furth, S. Pickup, E. S. Siegelman, and E. J. Delikatny, "In vivo assessment of hepatic triglycerides in murine non-alcoholic fatty liver disease using magnetic resonance spectroscopy," Biochimica et Biophysica Acta, vol. 1791, no. 8, pp. 757-763, 2009.

[42] J. Araya, R. Rodrigo, L. A. Videla et al., "Increase in long-chain polyunsaturated fatty acid n-6/n-3 ratio in relation to hepatic steatosis in patients with non-alcoholic fatty liver disease," Clinical Science, vol. 106, no. 6, pp. 635-643, 2004.

[43] L. A. Videla, R. Rodrigo, J. Araya, and J. Poniachik, "Oxidative stress and depletion of hepatic long-chain polyunsaturated fatty acids may contribute to nonalcoholic fatty liver disease," Free Radical Biology and Medicine, vol. 37, no. 9, pp. 1499-1507, 2004.

[44] J. H. Song, K. Fujimoto, and T. Miyazawa, "Polyunsaturated (n3) fatty acids susceptible to peroxidation are increased in plasma and tissue lipids of rats fed docosahexaenoic acid-containing oils," Journal of Nutrition, vol. 130, no. 12, pp. 3028-3033, 2000.

[45] J. H. Song and T. Miyazawa, "Enhanced level of n-3 fatty acid in membrane phospholipids induces lipid peroxidation in rats fed dietary docosahexaenoic acid oil," Atherosclerosis, vol. 155, no. 1, pp. 9-18, 2001.

[46] P. Puri, R. A. Baillie, M. M. Wiest et al., "A lipidomic analysis of nonalcoholic fatty liver disease," Hepatology, vol. 46, no. 4, pp. 1081-1090, 2007.

[47] C. P. F. Marinangeli and P. J. H. Jones, "Functional food ingredients as adjunctive therapies to pharmacotherapy for treating disorders of metabolic syndrome," Annals of Medicine, vol. 42, no. 5, pp. 317-333, 2010.

[48] A. Sahebkar, "Why it is necessary to translate curcumin into clinical practice for the prevention and treatment of metabolic syndrome?" Biofactors, vol. 39, no. 2, pp. 197-208, 2012. 


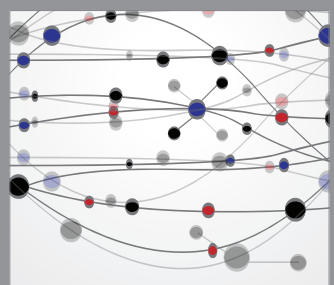

The Scientific World Journal
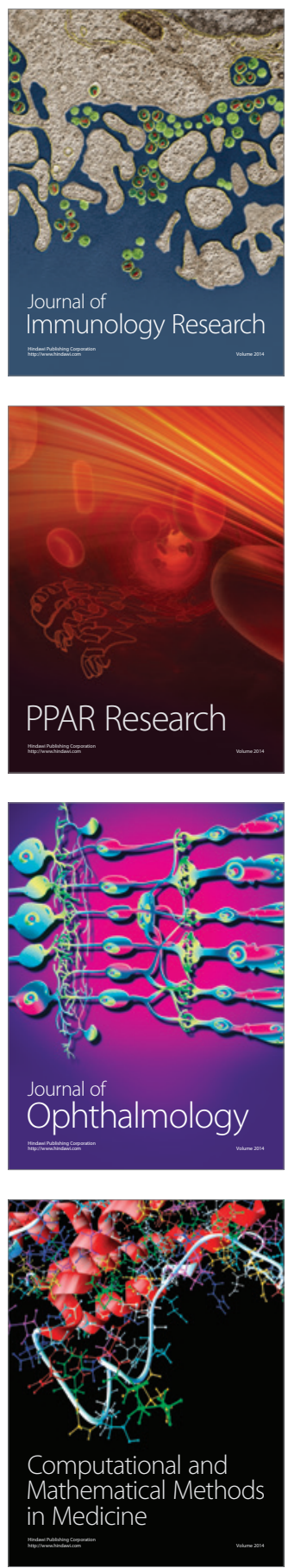

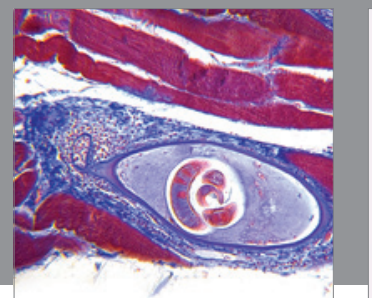

Gastroenterology

Research and Practice


\section{Hindawi}

Submit your manuscripts at

http://www.hindawi.com
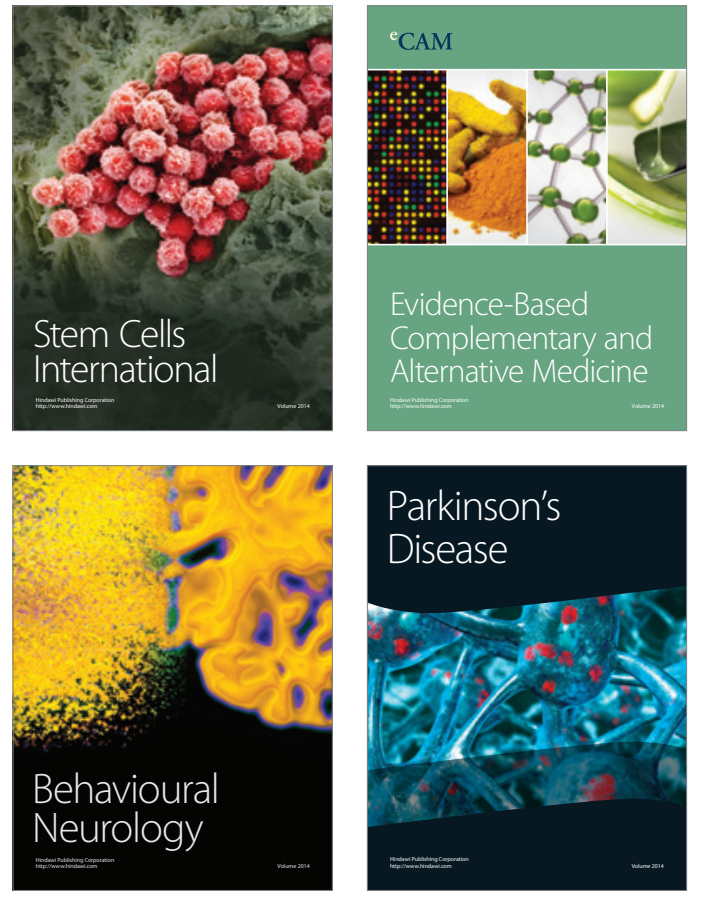
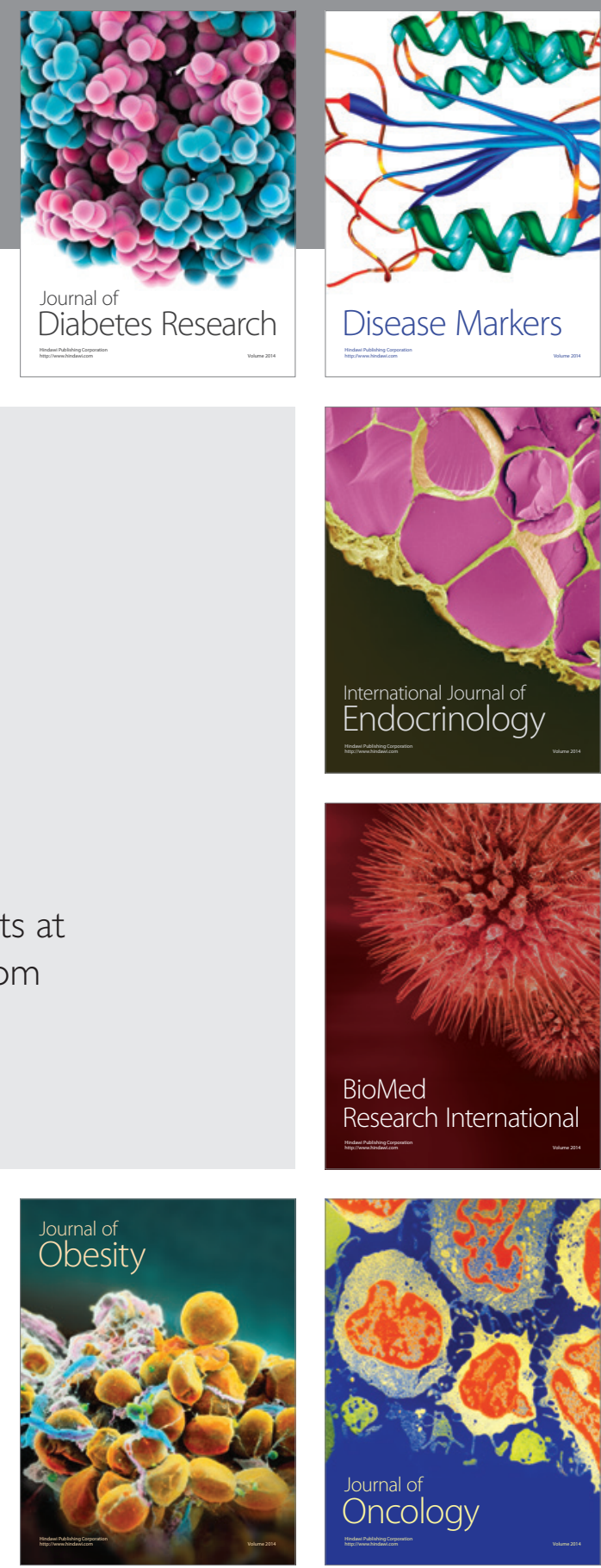

Disease Markers
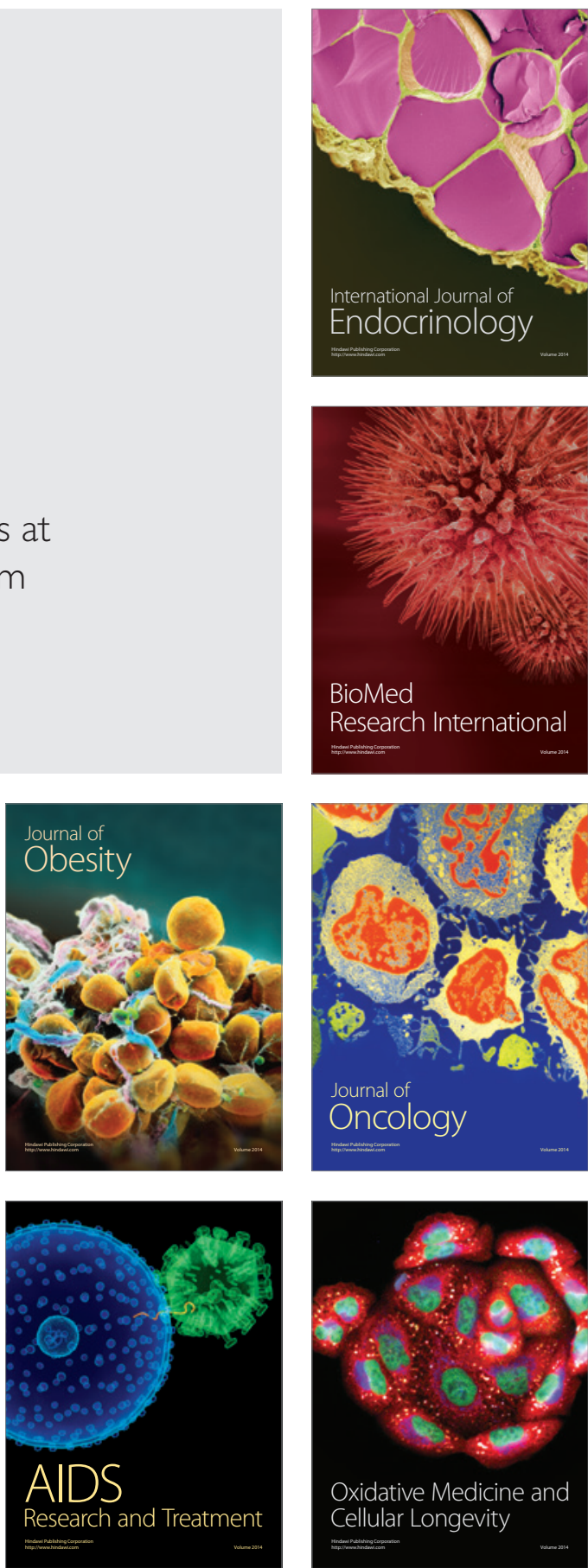